\title{
Venous Thromboembolism Prophylaxis and Hormonal Contraceptive Management Practice Patterns in the Perioperative Period for Anterior Cruciate Ligament Reconstruction
}

\author{
Robert A. Christian, M.D., M.B.A., Sarah T. Lander, M.D., \\ Nicholas A. Bonazza, M.D., M.H.A., Emily K. Reinke, Ph.D., \\ Trevor A. Lentz, P.T., Ph.D., M.P.H., Julie A. Dodds, M.D., Mary K. Mulcahey, M.D., \\ Anne C. Ford, M.D., and Jocelyn R. Wittstein, M.D.
}

\begin{abstract}
Purpose: To evaluate the venous thromboembolism (VTE) prophylaxis practices of surgeons performing anterior cruciate ligament reconstruction (ACLR) in female patients using hormonal contraceptives. Methods: Our research team designed an investigational survey using branching logic that was made available to the AANA membership. The survey was designed to identify clinical decision making regarding VTE prophylaxis after ACLR in patients without risk factors for VTE, the counseling of patients about VTE risk associated with hormonal contraceptives, and the use of VTE prophylaxis after ACLR in patients taking hormonal contraceptives. Results: Ninety-four respondents completed the survey. Eightynine respondents identified their gender (63\% male and 37\% female respondents). Respondents reported performing the following number of ACLRs annually: more than $50(40 \%), 30$ to $50(29 \%), 15$ to $30(29 \%)$, and fewer than $15(2 \%)$. Of the respondents, $62(67 \%)$ reported that VTE developed after ACLR in their patients (male patients only, 32\%; female patients only, 24\%; and both male and female patients, 34\%). Sixty-seven percent used chemoprophylaxis after ACLR. Surgeons who asked about hormonal contraceptive use were more likely to be women $(P=.01$; odds ratio [OR], 4.2$)$. Surgeons who changed their VTE prophylaxis plan as a result of asking about hormonal contraceptive use were more likely to be women $(P=.02 ; \mathrm{OR}, 2.8)$. Surgeons who asked about hormonal contraceptive use were more likely to have female patients with VTE after $\operatorname{ACLR}(P=.03 ; \mathrm{OR}, 2.9)$. Surgeons who changed their VTE prophylaxis plan as a result of asking about hormonal contraceptive use were more likely to have female patients with VTE after ACLR $(P=.001$; OR, 4.6). Conclusions: There is no standard of care for VTE prophylaxis after ACLR. A surgeon's own gender and prior clinical experience with VTE after ACLR may influence his or her likelihood to consider a patient's hormonal contraceptive use regarding VTE risk after ACLR. Clinical Relevance: The use of hormonal contraception is a risk factor for VTE in female patients undergoing ACLR. It is important to identify current practice patterns and the need for a standard of care.
\end{abstract}

$\mathbf{V}$ enous thromboembolism (VTE) is a rare but potentially fatal complication after anterior cruciate ligament reconstruction (ACLR). ${ }^{1-3}$ For arthroscopic

From the Department of Orthopaedic Surgery, Duke University, Durham, North Carolina, U.S.A. (R.A.C., S.T.L., N.A.B., E.K.R., T.A.L., J.R.W.); Duke Clinical Research Institute, Duke University, Durham, North Carolina, U.S.A. (T.A.L.); Department of Orthopaedic Surgery, Michigan State University, East Lansing, Michigan, U.S.A. (J.A.D.); Department of Orthopaedic Surgery, Tulane University, New Orleans, Louisiana, U.S.A. (M.K.M.); and Department of Obstetrics and Gynecology, Duke University, Durham, North Carolina, U.S.A. (A.C.F.).

The authors report the following potential conflicts of interest or sources of funding: M.K.M. is a consultant for courses for Arthrex, outside the submitted work. Full ICMJE author disclosure forms are available for this article online, as supplementary material. surgery and ACLR, postoperative VTE rates vary in the literature owing to numerous factors such as the method of detection, procedure performed, and use of

This paper was presented at the American Orthopaedic Society for Sports Medicine-AANA Combined 2021 Annual Meeting.

Received September 1, 2021; accepted December 1, 2021.

Address correspondence to Robert A. Christian, M.D., M.B.A., $622 \mathrm{~W} 168$ th St, PH-11-1130, New York, NY 10032,U.S.A. E-mail: rob.christian13@ gmail.com

(C) 2022 THE AUTHORS. Published by Elsevier Inc. on behalf of the Arthroscopy Association of North America. This is an open access article under the CC BY-NC-ND license (http://creativecommons.org/licenses/by-nc-nd/4.0/).

2666-061X/211241

https://doi.org/10.1016/j.asmr.2021.12.010 
prophylaxis, if any. Rates of deep vein thrombosis (DVT) and pulmonary embolism (PE) have been reported to range from $0.25 \%$ to $1.03 \%$ and from $0.05 \%$ to $0.19 \%$, respectively. ${ }^{4-9}$ The use of VTE prophylaxis after ACLR also varies because the literature has not clearly supported the routine use of chemoprophylaxis postoperatively. Comprehensive clinical guidance to help surgeons decide when such prophylaxis may be beneficial, particularly in patients at increased risk, is needed. ${ }^{10-12}$

Recent evidence has suggested a possible protective association between the use of hormonal contraceptives and anterior cruciate ligament injury as well as reinjury after reconstruction. ${ }^{13-15}$ On the other hand, the use of hormonal contraceptives is a known risk factor for thromboembolic events and, in ACLR, increases the risk of such events beyond the risk presented by surgery and rehabilitation alone. ${ }^{8}$ Both ACLR and the use of hormonal contraception are common in the young female population; hence, there is potential for cumulative risk of VTE. It is unclear whether surgeons regularly counsel patients on the risk of thromboembolic events associated with hormonal contraceptives prior to pursuing ACLR. Additionally, the management of such patients likely presents significant variability given the lack of clear clinical guidelines.

The purpose of this study was to evaluate the VTE prophylaxis practices of surgeons performing ACLR in female patients using hormonal contraceptives. Our hypothesis was that there would not be a standard of care among surgeons; however, some surgeons may change their management based on the presence of this risk factor and counsel patients on the risk of VTE associated with the use of hormonal contraceptives and surgery.

\section{Methods}

The survey (Appendix Fig 1, available at www. arthroscopyjournal.org) used in this study was developed collaboratively by sports medicine-trained, orthopaedic surgeons from 3 academic centers in conjunction with specialists in obstetrics and gynecology and was approved by the institutional review board. The survey was subsequently approved by the AANA Research Committee and was made available to the AANA membership for response. The survey was posted on the AANA website as a research survey; members could complete the survey at their leisure. The survey used branching logic and was designed to identify the respondent's clinical decision making regarding the use of VTE prophylaxis after ACLR in patients without risk factors for VTE, the counseling of patients about the risk of VTE associated with hormonal contraceptive use during the perioperative period, and the use of VTE prophylaxis after ACLR in patients taking hormonal contraceptives and whether it varies with delivery method (oral, vaginal, or transdermal). Questions also aimed to identify common practices of surgeons pertaining to use, type, timing, and length of VTE prophylaxis around surgery. The survey included questions on whether the surgeon routinely inquired about hormonal contraceptive use and whether the patient's answers changed the surgeon's prophylaxis treatment protocol. Pertaining to hormonal contraceptives, surgeons were asked whether they have patients stop taking hormonal contraceptives perioperatively, prescribe prophylaxis, or both. To further assess possible bias, surgeon demographic characteristics were identified including gender, age, geographic location, type of practice, and number of ACLRs performed per year. The survey also inquired whether respondents had prior patients in whom a VTE event developed after ACLR. The survey was administered and data were collected using REDCap. ${ }^{16,17}$

\section{Statistical Analysis}

Data were analyzed using the Kruskal-Wallis test to evaluate differences between groups and binary logistic regression to report odds ratios (ORs) associated with reporting dichotomous response options. For the purposes of regression analysis, we dichotomized responses regarding whether surgeons ask about hormonal contraceptive use (never, rarely, or sometimes vs frequently or always) and whether surgeons change their prophylaxis plan as a result of the information (no vs depends or yes). Microsoft Excel software (Redmond, WA) was used to summarize the responses to all survey questions and present descriptive statistics.

\section{Results}

A total of 94 AANA members completed the survey. One incomplete survey was received and was not included in the results. Of the member respondents, 56 were men $(62.9 \%), 33$ were women $(37.1 \%)$, and 5 did not provide their gender. The age of the respondents was as follows: $15.7 \%(\mathrm{n}=14)$ were younger than 40 years, $43.8 \%(n=39)$ were aged 40 to 49 years, $23.6 \%(\mathrm{n}=21)$ were aged 50 to 59 years, and $16.9 \%(n=15)$ were aged 60 years or older. The number of ACLRs performed annually by respondents was as follows: $40.4 \%(\mathrm{n}=38)$ performed more than 50 ACLRs, $28.7 \%(\mathrm{n}=27)$ performed 30 to $50,28.7 \%$ $(\mathrm{n}=27)$ performed 15 to 30 , and $2.1 \%(\mathrm{n}=2)$ performed fewer than 15 . The respondents reported working in the following practice settings: academic institution, $40.4 \%(\mathrm{n}=36)$; private practice, $32.6 \%$ $(\mathrm{n}=29)$; hospital employed, $22.5 \%(\mathrm{n}=20)$; military, $1.1 \%(\mathrm{n}=1)$; and other practice type, $3.4 \%(\mathrm{n}=3)$.

\section{Current Practice Patterns}

Of the respondents, $67 \%(n=62)$ routinely use pharmacologic VTE prophylaxis postoperatively in 
patients after ACLR. Ninety-eight percent of respondents $(\mathrm{n}=61)$ identified "reduce risk of DVT/PE" as a reason for using pharmacologic VTE prophylaxis. Thirty-seven percent of respondents $(\mathrm{n}=23)$ identified "medical-legal concerns" as a reason for using pharmacologic VTE prophylaxis.

Regarding VTE prophylaxis regimens, 97\% of respondents $(n=60)$ using pharmacologic prophylaxis reported routinely using an aspirin-based regimen. Of these respondents, $61 \%(n=37)$ reported using an aspirin-based regimen at a dose of $325 \mathrm{mg}$ whereas $35 \%(\mathrm{n}=21)$ reported using an aspirin-based regimen at a dose of $81 \mathrm{mg}$. One respondent reported using a naproxen-based regimen at a dose of $500 \mathrm{mg}$. One respondent reported using an Eliquis-based regimen (Bristol-Myers Squibb, New York, NY) at a dose of 5 mg. The most common regimen used among respondents was $325 \mathrm{mg}$ of aspirin daily for 14 days (15\% of respondents, $\mathrm{n}=9$ ).

Regarding the duration of VTE prophylaxis after surgery, the most common duration was 14 days postoperatively $(40 \%, \mathrm{n}=25)$ (Fig 1). Ninety-eight percent of respondents $(\mathrm{n}=61)$ prescribed prophylaxis for at least 14 days; $58 \%$ of respondents $(\mathrm{n}=37)$, at least 21 days; and $47 \%$ of respondents $(\mathrm{n}=30)$, at least 28 days.

When we evaluated whether respondents had prior patients in whom VTE developed after ACLR, 67\% of respondents $(n=62)$ reported that they had this prior personal experience. Of these respondents, $32 \%(\mathrm{n}=$ 20 ) had only male patients with VTE after ACLR; $24 \%$ $(\mathrm{n}=15)$, only female patients; and $33 \%(\mathrm{n}=21)$, both male and female patients. Thus, $57 \%(\mathrm{n}=36)$ reported clinical experience with female patients who had VTE after ACLR whereas $32 \%(n=20)$ reported no exposure to female patients with VTE after ACLR.

When considering female patients, $40 \%$ of surgeons $(\mathrm{n}=37)$ responded that they always inquire about hormonal contraceptive medications, $27 \%(\mathrm{n}=25)$ frequently ask, $9 \%(\mathrm{n}=8)$ sometimes ask, $14 \%(\mathrm{n}=$ 13) rarely ask, and $10 \%(n=9)$ never ask. When considering the reasons respondents may not ask patients about the use of hormonal contraceptive medications, $63.7 \%(\mathrm{n}=37)$ indicated that it does not change the treatment plan, $14.5 \%(\mathrm{n}=8)$ believe the risk of blood clots associated with hormonal contraceptives is low, $18.2 \%(\mathrm{n}=10)$ adjust perioperative prophylaxis based on medications listed in the medical record, $5.5 \%(\mathrm{n}=3)$ desire to avoid an uncomfortable subject, $9.1 \%(\mathrm{n}=5)$ indicated that the parents of a minor patient are present, $1.8 \%(\mathrm{n}=1)$ would not ask if they did not think the patient had a reason to be receiving hormonal contraceptives, and $10.9 \%(n=6)$ reported other reasons.

When considering whether hormonal contraceptive use changes the care plan in female patients

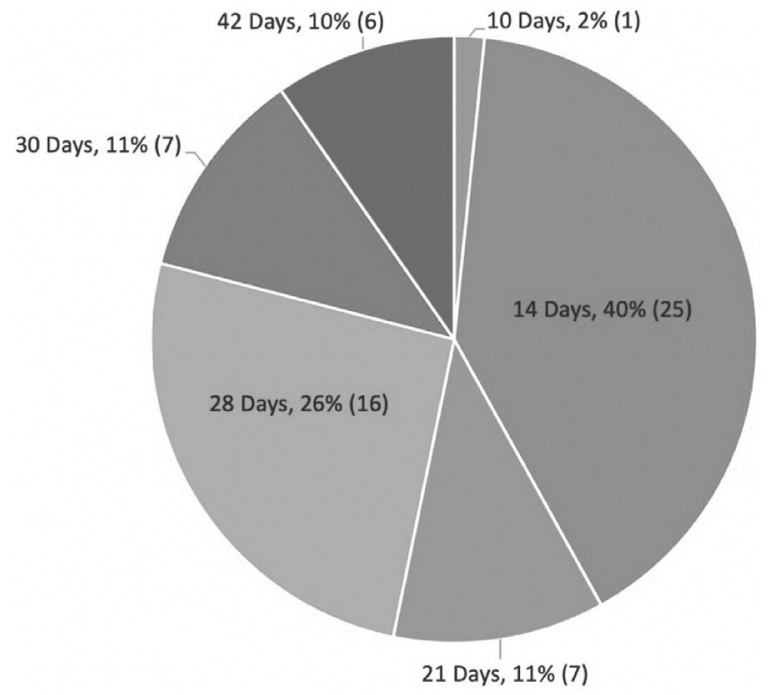

Fig 1. Pie chart showing various durations of routine venous thromboembolism prophylaxis use among respondents.

undergoing ACLR, 55\% of respondents $(n=51)$ responded "no"; $33 \%(\mathrm{n}=30)$ responded "yes, but I change my care plan in the same way regardless of type of hormonal contraceptive"; and $12 \%(\mathrm{n}=11)$ responded "depends on the type of hormonal contraceptive." Of those respondents who reported "yes, but I change my care plan in the same way regardless of type of hormonal contraceptive" in response to the question on hormonal contraceptive use in female patients, $37 \%$ $(\mathrm{n}=11)$ reported "prescribing pharmacologic prophylaxis even though I do NOT do so routinely"; $30 \%$ (n = 9) reported "asking the patient to stop hormonal contraceptive perioperatively"; $23 \%(\mathrm{n}=7)$ reported "using different pharmacologic prophylaxis"; $10 \%(\mathrm{n}=3)$ reported "increasing duration of routine pharmacologic prophylaxis"; and 7\% $(\mathrm{n}=2)$ reported "increasing dose of routine pharmacologic prophylaxis." Those respondents who reported "depends on the type of hormonal contraceptive" in response to the question regarding hormonal contraceptive use in female patients were more likely to ask a patient to stop taking oral contraceptive pills (OCPs) in the perioperative period rather than to remove a transdermal patch or vaginal ring. Respondents were also more likely to prescribe pharmacologic VTE prophylaxis, even if they do not do so routinely after ACLR, in a patient taking OCPs rather than a patient with a transdermal patch or vaginal ring.

Surgeons who frequently or always asked about hormonal contraceptive use were more likely to be women $(P=.01 ; \mathrm{OR}, 4.2)$ (Table 1$)$, and those who changed their VTE prophylaxis plan as a result of asking about hormonal contraceptive use (reporting yes or depends) were also more likely to be women $(P=.02$; OR, 2.8) (Table 2). Surgeons who frequently or always 
Table 1. Percentage of Surgeons Who Always or Frequently Ask About Hormonal Contraceptive Use

\begin{tabular}{lrrr}
\hline & \multicolumn{3}{c}{ Always or Frequently Ask About DVT Prophylaxis } \\
\cline { 2 - 4 } & Female Surgeons & Male Surgeons & All Surgeons \\
\hline Overall & $85 \%(28 \text { of } 33)^{*}$ & $57 \%(32 \text { of } 56)^{*}$ & \\
History of female patient with DVT & & & \\
Yes & $100 \%(11$ of 11$)$ & $71 \%(17$ of 24$)$ & of 89$)$ \\
No & $77 \%(17$ of 22$)$ & $47 \%(15$ of 32$)$ & $50 \%(28 \text { of } 35)^{\dagger}$ \\
\hline
\end{tabular}

DVT, deep vein thrombosis.

*Statistically significant difference in proportions between groups: $P=.01$.

${ }^{\dagger}$ Statistically significant difference in proportions between groups: $P=.03$.

asked about hormonal contraceptive use were more likely to have had a female patient with VTE after ACLR $(P=.03 ;$ OR, 2.9) (Table 1), and those who changed their VTE prophylaxis plan as a result of asking about hormonal contraceptive use (reporting yes or depends) were more likely to have had a female patient with VTE after $\operatorname{ACLR}(P=.001 ;$ OR, 4.6) (Table 2).

\section{Discussion}

The survey results in this study show that prior clinical experience with VTE after ACLR and surgeon gender play significant roles in orthopaedic surgeons' management of VTE risk after ACLR. Two-thirds of our respondents reported having a prior patient in whom VTE developed after ACLR. When considering female patients for ACLR, $40.2 \%$ of survey respondents reported asking about hormonal contraceptive use. When stratifying surgeons by gender, surgeons who always or frequently asked patients about hormonal contraceptive use and surgeons who changed their VTE prophylaxis plan as a result of asking about hormonal contraceptive use were 4.2 and 2.8 times more likely to be women, respectively $(P=.01$ and $P=.02$, respectively). The role of surgeon gender in asking about hormonal contraceptive use and adjusting the VTE prophylaxis plan may relate to increased awareness of hormonal contraceptive medications as a risk factor for VTE, as well as personal experience with hormonal contraceptive medications. Surgeons who frequently or always asked about hormonal contraceptive use and those who changed their VTE prophylaxis plan as a result of asking about hormonal contraceptive use were
2.9 and 4.6 times more likely to have had prior experience with a female patient with VTE after ACLR, respectively $(P=.03$ and $P=.001$, respectively). This finding suggests that prior clinical experience likely plays a significant role in surgeons considering and adjusting hormonal contraceptive use and the VTE plan in the perioperative period after ACLR.

The use of VTE prophylaxis after ACLR varies across surgeons. A 2018 study by Keller et al. ${ }^{10}$ found that over half of 142 orthopaedic sports medicine fellowship-trained surgeons in the United States use chemoprophylaxis after ACLR-with over $90 \%$ of surgeons who use chemoprophylaxis prescribing aspirin. The findings of this study align with our survey results, with $66.7 \%$ of respondents using VTE chemoprophylaxis after ACLR and $97 \%$ of these respondents using an aspirin-based regimen. Keller et al. also reported that $44 \%$ of surgeons who do not routinely use chemoprophylaxis said that they would consider its use in patients who currently use hormonal contraception. In a recent survey of 115 surgeons performing ACLR in Sweden, Ekdahl et al. ${ }^{18}$ found that only $16 \%$ of surgeons always prescribed thromboprophylaxis but $82 \%$ prescribed it in patients using oral contraceptives, second only to patients with a history of thrombosis $(99 \%)$.

Fortunately, the risk factors for perioperative thromboembolic disease have been well studied. Known risk factors for VTE include increasing age, cancer, smoking, obesity, thrombophilia, chronic venous insufficiency, use of hormonal contraceptive medications, family history of VTE, and personal history of VTE. ${ }^{5,13,19,20}$

Table 2. Percentage of Surgeons Who Always or Frequently Change DVT Prophylaxis Based on Patient History of DVT

\begin{tabular}{|c|c|c|c|}
\hline & \multicolumn{3}{|c|}{ Always or Frequently Change DVT Prophylaxis } \\
\hline Overall & $61 \%(20 \text { of } 33)^{*}$ & $36 \%(20 \text { of } 56)^{*}$ & $44 \%(40$ of 89$)$ \\
\hline Yes & $73 \%(8$ of 11$)$ & $63 \%(15$ of 24$)$ & $66 \%(23$ of 35$)$ \\
\hline No & $55 \%(12$ of 22$)$ & $16 \%(5$ of 32$)$ & $31 \%(17$ of 54$)$ \\
\hline
\end{tabular}

DVT, deep vein thrombosis.

*Statistically significant difference in proportions between groups: $P=.02$.

${ }^{\dagger}$ Statistically significant difference in proportions between groups: $P=.001$. 
A recent database study by Traven et al. ${ }^{5}$ expanded on prior studies on hormonal contraceptives to look specifically at the risk of VTE associated with combined oral contraceptive pills (COCPs). The authors reported that COCP use increases the risk of symptomatic DVT and PE after knee arthroscopy and the risk of DVT-but not PE-after ACLR. The OR of any DVT or PE after ACLR with the use of COCPs was noted to be $2.34(P<.001)$.

Postoperative VTE prophylaxis management is a multifaceted decision for orthopaedic surgeons weighing multiple relative risks including baseline VTE risk, postoperative bleeding concerns, procedure-related factors, and anesthesia-related factors (regional blocks or catheter use), as well as patient-related risk factors. Currently, we are not aware of an organization or society that recommends the routine use of chemoprophylaxis to prevent thromboembolic events after non-arthroplasty orthopaedic surgery, and the American College of Chest Physicians (ACCP) recommends against such prophylaxis unless a patient has a history of DVT. ${ }^{12}$ Several studies have looked at the evidence for chemoprophylaxis in patients undergoing knee arthroscopy. In 2008, a Cochrane Review by Ramos et al. ${ }^{21}$ found that the relative risk of thrombotic events was 0.16 when using low-molecular-weight heparin (LMWH) in patients undergoing knee arthroscopy. In 2014, Chapelle et al. ${ }^{22}$ included 14 studies in their meta-analysis of patients requiring leg immobilization for non-major orthopaedic injuries or undergoing knee arthroscopy and found a $68 \%$ reduction in the risk of major VTE events $(P<.001)$ in patients receiving LMWH-although they also observed a nonsignificant $35 \%$ increase in the risk of major bleeding. Both studies, however, noted the significant lack of consistency in data particularly regarding the stratification of procedures being performed in the various studies. More recently, in 2019, a systematic review and metaanalysis by Zhu et al. ${ }^{23}$ reported that LMWH was effective in decreasing VTE in patients undergoing ACLR, and although there was an increase in minor complications, there was no increased risk of clinically relevant major bleeding complications. Additionally, a 2020 Cochrane Review by Perrotta et al. ${ }^{24}$ compared LMWH with compression stockings, rivaroxaban, aspirin, and placebo and found moderate- to lowcertainty evidence of no benefit from the use of chemoprophylaxis in healthy patients while finding no differences in adverse events between groups. Although evidence has supported the common use of aspirin for chemoprophylaxis after total hip and knee arthroplasty, there is little evidence to guide its use in patients undergoing ACLR. ${ }^{25,26}$ Overall, the literature suggests that VTE prophylaxis is effective in decreasing rates of VTE, but the complexity of endogenous patient VTE risks, exogenous VTE risks, postoperative bleeding concerns, and other factors complicate clinical decision making. Further research is needed to determine which patients, especially those at increased VTE risk, would benefit from postoperative prophylaxis, as well as the optimal chemoprophylactic regimen.

Oral contraceptives are the most-used reversible form of contraception in the United States, and as a result, orthopaedic surgeons treating female patients aged 15 to 49 years are likely to encounter patients using these drugs. ${ }^{27}$ An understanding of the inherent clotting risks related to the use of these medications, as well as the different formulations, and counseling of patients regarding postoperative thromboembolic risks should be part of routine preoperative counseling for women using these medications.

Oral contraceptive medications have multiple formulations that can be simplified into 2 groups: (1) progestin only or (2) estrogen and progestin in combination (also called "combined oral contraceptives"). The estrogenic component of combined oral contraceptives increases the risk of VTE by increasing hepatic production of serum globulins involved in coagulation. Progestin-only pills do not carry the same thromboembolic risk as combined oral contraceptives. Combination birth control "pills" are now available in transdermal and transvaginal delivery systems, bypassing the need for daily oral dosing. The clotting risks of these formulations should be considered the same as those of the oral formulations. ${ }^{28-30}$

In addition to the risks of surgery itself, the use of combined oral contraceptives is associated with a 2- to 6-fold relative risk of VTE. Compared with nonpregnant, non-hormone users, the risk of VTE for combined oral contraceptive users increases from 1 to 5 per 10,000 woman-years of use to 3 to 9 per 10,000 woman-years of use. This risk is still about half the VTE risk of pregnancy (5 to 20 per 10,000 woman-years) and still lower than the VTE risk in the postpartum period (40 to 65 per 10,000 woman-years). ${ }^{31}$

Counseling women on DVT risk reduction prior to surgery is complicated, balancing the risk of unintended pregnancy against the compounded risk of hormonal contraceptive use and major surgery. No clear consensus exists for specific pharmacologic prevention of VTE in women who continue using hormonal contraceptives while undergoing major surgery. The Centers for Disease Control and Prevention, the World Health Organization, and the UK Medical Eligibility Criteria recommend discontinuing OCPs in women undergoing major surgery with prolonged immobilization but are divided on whether to continue OCPs in women undergoing major surgery without prolonged immobilization. $^{30}$

Overall, the aim of our study was to determine the management practices and use of VTE prophylaxis among surgeons performing ACLR in female patients using hormonal contraceptives. A secondary goal of this 
study was to raise awareness and identify potential knowledge gaps in practice patterns related to VTE risk in this area of common clinical intersection: female patients who are taking hormonal contraceptive medications and undergoing ACLR. The results of this study do not show a clear standard of care among orthopaedic sports medicine surgeons and should not be used to identify any malpractice. This study highlights the importance of recognizing VTE risk associated with ACLR and various risk factors, including hormonal contraceptive use. Further research is needed to raise awareness among surgeons, to identify which management practices best decrease VTE risk in our patients, and hopefully, to develop clinical guidelines to direct practice.

\section{Limitations}

There are potential weaknesses of this study that should be acknowledged. First, our survey queried the AANA membership, which may introduce selection bias and limit the generalizability to overall national practice patterns across the United States. Second, there may be a response bias such that surgeons responding to this survey have greater interest in the topic and possibly greater knowledge of the topic as compared with surgeons who did not respond. This likely leads to an overestimation of the number of providers who routinely ask about hormonal contraceptive use or change their practice based on the use of contraceptive medications compared with national practice patterns. Additionally, because the survey was posted on the AANA website and because all members were not directly queried, the number of members who may not have completed the survey or been aware of it is unknown. Finally, the results of the survey represent the opinions and recollections of our survey respondents and cannot be verified with clinical data.

\section{Conclusions}

There is no standard of care for VTE prophylaxis after ACLR. A surgeon's own gender and prior clinical experience with VTE after ACLR may influence his or her likelihood to consider a patient's hormonal contraceptive use regarding VTE risk after ACLR.

\section{Acknowledgment}

The authors thank the AANA Research Committee for its assistance with this study.

\section{References}

1. Bourget-Murray J, Clarke MA, Gorzitza S, Phillips LA. Symptomatic bilateral pulmonary embolism without deep venous thrombosis in an adolescent following arthroscopic anterior cruciate ligament reconstruction: A case report and review of the literature. I Med Case Rep 2018;12:194.

2. Gaskill T, Pullen M, Bryant B, Sicignano N, Evans AM, DeMaio M. The prevalence of symptomatic deep venous thrombosis and pulmonary embolism after anterior cruciate ligament reconstruction. Am J Sports Med 2015;43: 2714-2719.

3. Janssen RP, Sala HA. Fatal pulmonary embolism after anterior cruciate ligament reconstruction. Am J Sports Med 2007;35:1000-1002.

4. Abram SGF, Judge A, Beard DJ, Price AJ. Rates of adverse outcomes and revision surgery after anterior cruciate ligament reconstruction: A study of 104,255 procedures using the National Hospital Episode Statistics Database for England, UK. Am J Sports Med 2019;47:2533-2542.

5. Traven SA, Farley KX, Gottschalk MB, et al. Combined oral contraceptive use increases the risk of venous thromboembolism after knee arthroscopy and anterior cruciate ligament reconstruction: An analysis of 64,165 patients in the Truven database. Arthroscopy 2021;37: 924-931.

6. Ilahi OA, Reddy J, Ahmad I. Deep venous thrombosis after knee arthroscopy: A meta-analysis. Arthroscopy 2005;21:727-730.

7. Kraus Schmitz J, Lindgren V, Janarv PM, Forssblad M, Stalman A. Deep venous thrombosis and pulmonary embolism after anterior cruciate ligament reconstruction: Incidence, outcome, and risk factors. Bone Joint J 2019;101-B:34-40.

8. Maletis GB, Inacio MC, Reynolds S, Funahashi TT. Incidence of symptomatic venous thromboembolism after elective knee arthroscopy. J Bone Joint Surg Am 2012;94: 714-720.

9. Mauck KF, Froehling DA, Daniels PR, et al. Incidence of venous thromboembolism after elective knee arthroscopic surgery: A historical cohort study. J Thromb Haemost 2013;11:1279-1286.

10. Keller RA, Moutzouros V, Dines JS, Bush-Joseph CA, Limpisvasti O. Deep venous thrombosis prophylaxis in anterior cruciate ligament reconstructive surgery: What is the current state of practice? Sports Health 2018;10: 156-159.

11. van Adrichem RA, Nemeth B, Algra A, et al. Thromboprophylaxis after knee arthroscopy and lower-leg casting. N Engl J Med 2017;376:515-525.

12. Falck-Ytter Y, Francis CW, Johanson NA, et al. Prevention of VTE in orthopedic surgery patients: Antithrombotic Therapy and Prevention of Thrombosis, 9th ed: American College of Chest Physicians Evidence-Based Clinical Practice Guidelines. Chest 2012;141:e278S-e325S.

13. Rahr-Wagner L, Thillemann TM, Mehnert F, Pedersen $A B$, Lind $M$. Is the use of oral contraceptives associated with operatively treated anterior cruciate ligament injury? A case-control study from the Danish Knee Ligament Reconstruction Registry. Am J Sports Med 2014; 42:2897-2905.

14. Samuelson K, Balk EM, Sevetson EL, Fleming BC. Limited evidence suggests a protective association between oral contraceptive pill use and anterior cruciate ligament injuries in females: A systematic review. Sports Health 2017;9:498-510. 
15. Gray AM, Gugala Z, Baillargeon JG. Effects of oral contraceptive use on anterior cruciate ligament injury epidemiology. Med Sci Sports Exerc 2016;48:648-654.

16. Harris PA, Taylor R, Minor BL, et al. The REDCap consortium: Building an international community of software platform partners. J Biomed Inform 2019;95:103208.

17. Harris PA, Taylor R, Thielke R, Payne J, Gonzalez N, Conde JG. Research electronic data capture (REDCap) - A metadata-driven methodology and workflow process for providing translational research informatics support. J Biomed Inform 2009;42:377-381.

18. Ekdahl V, Stalman A, Forssblad M, Samuelsson K, Edman G, Kraus Schmitz J. There is no general use of thromboprophylaxis and prolonged antibiotic prophylaxis in anterior cruciate ligament reconstruction: A nationwide survey of ACL surgeons in Sweden. Knee Surg Sports Traumatol Arthrosc 2020;28:2535-2542.

19. Delis KT, Hunt N, Strachan RK, Nicolaides AN. Incidence, natural history and risk factors of deep vein thrombosis in elective knee arthroscopy. Thromb Haemost 2001;86: 817-821.

20. Krych AJ, Sousa PL, Morgan JA, Levy BA, Stuart MJ, Dahm DL. Incidence and risk factor analysis of symptomatic venous thromboembolism after knee arthroscopy. Arthroscopy 2015;31:2112-2118.

21. Ramos J, Perrotta C, Badariotti G, Berenstein G. Interventions for preventing venous thromboembolism in adults undergoing knee arthroscopy. Cochrane Database Syst Rev 2008:CD005259.

22. Chapelle C, Rosencher N, Jacques Zufferey P, et al. Prevention of venous thromboembolic events with lowmolecular-weight heparin in the non-major orthopaedic setting: Meta-analysis of randomized controlled trials. Arthroscopy 2014;30:987-996.

23. Zhu J, Jiang H, Marshall B, Li J, Tang X. Low-molecularweight heparin for the prevention of venous thromboembolism in patients undergoing knee arthroscopic surgery and anterior cruciate ligament reconstruction: A meta-analysis of randomized controlled trials. Am J Sports Med 2019;47:1994-2002.

24. Perrotta C, Chahla J, Badariotti G, Ramos J. Interventions for preventing venous thromboembolism in adults undergoing knee arthroscopy. Cochrane Database Syst Rev 2020;5:CD005259.

25. Anderson DR, Dunbar M, Murnaghan J, et al. Aspirin or rivaroxaban for VTE prophylaxis after hip or knee arthroplasty. N Engl J Med 2018;378:699-707.

26. Matharu GS, Kunutsor SK, Judge A, Blom AW, Whitehouse MR. Clinical effectiveness and safety of aspirin for venous thromboembolism prophylaxis after total hip and knee replacement: A systematic review and meta-analysis of randomized clinical trials. JAMA Intern Med 2020;180:376-384.

27. Kavanaugh ML, Pliskin E, Jerman J. Use of concurrent multiple methods of contraception in the United States, 2008 to 2015. Contracept X 2021;3:100060.

28. Jick SS, Hagberg KW, Hernandez RK, Kaye JA. Postmarketing study of ORTHO EVRA and levonorgestrel oral contraceptives containing hormonal contraceptives with 30 mcg of ethinyl estradiol in relation to nonfatal venous thromboembolism. Contraception 2010;81:16-21.

29. Raymond EG, Burke AE, Espey E. Combined hormonal contraceptives and venous thromboembolism: Putting the risks into perspective. Obstet Gynecol 2012;119:1039-1044.

30. Tepper NK, Dragoman MV, Gaffield ME, Curtis KM. Nonoral combined hormonal contraceptives and thromboembolism: A systematic review. Contraception 2017;95: $130-139$

31. FDA Drug Safety Communication: Updated information about the risk of blood clots in women taking birth control pills containing drospirenone. Silver Spring, MD: Food and Drug Administration, 2018. 